\title{
The effect of posterior compression of the facet joints for initial stability and sagittal profile in the treatment of thoracolumbar fractures: a biomechanical study
}

\author{
Michael Ruf ${ }^{1}$ - Tobias Pitzen ${ }^{1}$. Ivo Nennstiel ${ }^{2}$. David Volkheimer ${ }^{3}$. Jörg Drumm ${ }^{1} \cdot$ Klaus Püschel $^{4}$. \\ Hans-Joachim Wilke ${ }^{3}$ (D)
}

Received: 9 December 2020 / Revised: 1 August 2021 / Accepted: 11 October 2021 / Published online: 13 November 2021

(c) The Author(s) 2021

\begin{abstract}
Purpose Surgical treatment of thoracolumbar A3-fractures usually comprises posterior fixation-in neutral position or distraction-potentially followed by subsequent anterior support. We hypothesized that additional posterior compression in circumferential stabilization may increase stability by locking the facets, and better restore the sagittal profile.

Methods Burst fractures Type A3 were created in six fresh frozen cadaver spine segments (T12-L2). Testing was performed in a custom-made spinal loading simulator. Loads were applied as pure bending moments of $\pm 3.75 \mathrm{Nm}$ in all six movement axes. We checked range of motion, neutral zone and Cobb's angle over the injured/treated segment within the following conditions: Intact, fractured, instrumented in neutral alignment, instrumented in distraction, with cage left in posterior distraction, with cage with posterior compression.

Results We found that both types of instrumentation with cage stabilized the segment compared to the fractured state in all motion planes. For flexion/extension and lateral bending, flexibility was decreased even compared to the intact state, however, not in axial rotation, being the most critical movement axis. Additional posterior compression in the presence of a cage significantly decreased flexibility in axial rotation, thus achieving stability comparable to the intact state even in this movement axis. In addition, posterior compression with cage significantly increased lordosis compared to the distracted state. Conclusion Among different surgical modifications tested, circumferential fixation with final posterior compression as the last step resulted in superior stability and improved sagittal alignment. Thus, posterior compression as the last step is recommended in these pathologies.
\end{abstract}

Keywords Thoracolumbar fractures $\cdot$ Sagittal profile $\cdot$ Posterior compression $\cdot$ Stability $\cdot$ Biomechanical study

\section{Introduction}

Hans-Joachim Wilke

hans-joachim.wilke@uni-ulm.de

1 Center for Spine Surgery, Orthopedics, and Traumatology, SRH Klinikum Karlsbad-Langensteinbach, Guttmannstrasse 1, 76307 Karlsbad, Germany

2 Center for Orthopedic Surgery and Traumatology, SRH Central Hospital Suhl, Albert-Schweitzer-Strasse 2, 98527 Suhl, Germany

3 Institute of Orthopaedic Research and Biomechanics, University of Ulm, Helmholtzstrasse 14, $89901 \mathrm{Ulm}$, Germany

4 Department of Legal Medicine, University Hospital Hamburg-Eppendorf, Butenfeld 34, 22529 Hamburg, Germany
Spinal fractures at the thoracolumbar junction are the most frequent fractures within the thoracic and lumbar spine section. They account for $67 \%$ of these fractures with the first lumbar vertebra affected in most of the cases [1]. Compression fractures Type A according to Magerl's classification [2] predominate by far and account for $66 \%$ of all TL fractures.

Treatment for this entity depends on the type of injury, the degree of kyphosis, and neurological impairment. Surgery is recommended in case of instability, pronounced kyphosis, and/or encroachment of the spinal canal.

Currently, there is no standard procedure for surgical treatment of burst fractures Type A3 according to Magerl [2]. Single posterior instrumentations and fusions are 
performed as well as single anterior instrumentations and combined anterior-posterior stabilizations and fusions [1, 3-5].

Combined posterior-anterior stabilization provides a stable reconstruction with less implant failures and less loss of correction in the sagittal plane compared to a single posterior procedure $[1,6-9]$. In this procedure, the first step comprises a posterior instrumentation from the vertebra above the fractured vertebra to the vertebra below the fracture. This instrumentation is usually fixed in neutral position or in distraction to decompress the spinal canal via ligamentotaxis in case of encroachment [10]. In the second anterior approach the fractured vertebral body is replaced by a cage/spacer to restore the anterior load transmission [11].

Biomechanical in vitro studies ascertained that in posterior-anterior procedures the axial rotation is the most problematic movement axis with respect to stabilization. While in flexion/extension as well as in side bending the stability of the posterior-anterior construct is superior to the intact spine, it is different in axial rotation: stiffness of the posterior-anterior constructs with pedicle screws is reduced or at most similar to the intact spine $[12,13]$.

Slosar et al. performed an in vitro investigation using spines with simulated fractures to which different instrumentation types were applied. They found, that transpedicular constructs improved the stability of the injured spine beyond that of the intact spine in flexion and lateral bending at low loads. At high loads, they restored the stiffness to intact levels. However, in axial rotation they did not restore the stiffness to pre-injury level, particularly when the posterior column was disrupted. The authors recommended, that it may be necessary to augment the transpedicular construct, particularly when the posterior column is disrupted [14].

Posterior compression of the posterior aspect of the spine, i.e. compression via the pedicle screws as the final step of surgery, has been hypothesized to be beneficial with respect to initial stability particularly in axial rotation, and with respect to sagittal profile [15]. This hypothesis, however, has not yet been proven to the best knowledge of the authors.

Thus, the purpose of this biomechanical in vitro study was to evaluate if an additional posterior compression in a posterior-anterior (circumferential) procedure could improve initial stability when compared to the construct left in distraction.

Consequently, our hypotheses were:

1. Additional posterior compression in circumferential stabilization via the inserted pedicle screws increases initial stability compared to the construct left in distraction.

2. Additional posterior improves the correction of kyphosis by posterior shortening against the anterior spacer (cage) compared to the construct left in distraction.

\section{Materials and methods}

\section{Specimens and preparation}

Six fresh frozen cadaveric bisegmental human spine segments (T12-L2) with an age between 56 and 77 years and a bone mineral density between 66.1 and $105.9 \mathrm{mg} \mathrm{Ca}-\mathrm{Ha} /$ $\mathrm{cm}^{3}$ were used for biomechanical testing. The spines were taken from fresh cadavers during routine autopsies within the Institute of Forensic Medicine, University Hospital Hamburg-Eppendorf, Germany. The specimens were exclusively used for this study as described below and approved by ethic committee at the University of Ulm, Germany (46/13).

The specimens were sealed in triple plastic bags and frozen at $-20{ }^{\circ} \mathrm{C}$ until the experiment was done. They were thawed at $4{ }^{\circ} \mathrm{C}$ and prepared at room temperature. Soft tissues were removed; all ligaments, joint capsules, and discs were meticulously saved. The upper half of the cranial vertebra and the lower half of the caudal vertebra were embedded in polymethylmethacrylate (PMMA, Technovit 3040, Heraeus Kulzer, Wehrheim, Germany) [16]. After embedding, flanges were fixed to the PMMA blocks and during the tests, the specimens were kept moist with physiological saline of $0.9 \%$ [17].

\section{Testing protocol}

Flexibility tests were performed in a custom-made spinal loading simulator according to a generally accepted and standardized testing protocol [18]. The bisegmental specimens were loaded with pure moments of $\pm 3.75 \mathrm{Nm}$ without preload [16]. The load was applied continuously using three stepper motors (Isel 3450, Isert-electronic, Eiterfeld, Germany) at a loading rate of $1 \%$ (axial rotation $0.5 \%$ ) in the three principal motion planes-flexion/extension, lateral bending right/left and axial rotation left/right. During loading, the specimens were allowed to move unconstrained in the remaining five degrees of freedom. A total of 3.5 loading cycles was performed; the first two were used for preconditioning, and the third cycle was used for data analysis [16]. The moments were measured by a 6-DOF (degree of freedom) load cell (FT 1500/40, Schunk GmbH \& Co. KG, Lauffen/ Neckar, Germany, measuring error $<1 \%$ ). From the load-deformation curve, range of motion (ROM) and neutral zone (NZ) were determined.

The sagittal lordosis angle was measured bisegmentally from fluoroscope images by means of the Cobbmethod between the lower endplate of the vertebra above (Th12) and the upper endplate of the vertebra below ( $L 2)$. The sagittal angles were measured before and after each 
treatment step (Note-kyphosis angulation is labelled by a “+”, lordosis by a “-”).

Each of the specimens was tested in six conditions (Fig. 1):

1. Intact (Fig. 1a),

2. Fractured (specimen after creating a burst fracture A3 (Fig. 1b),

3. Instrumented with a pedicle screw-rod-system (USS Fracture System, Synthes GmbH, Oberdorf, Switzerland) in neutral position (after instrumentation in neutral position) (Fig. 1c),

4. Instrumented in distracted position ( $5 \mathrm{~mm}$ distraction, fixed screw/rod angle), simulating indirect decompression of the spinal canal via ligamentotaxis) (Fig. 1d),

5. insertion of a cage (expandable Synex cage $10^{\circ}$, Synthes $\mathrm{GmbH}$, Oberdorf, Switzerland), instrumentation unchanged (Fig. 1e),
6. Cage with posterior compression (compression of $120 \mathrm{~N}$ via the instrumentation with unfixed angulation) (Fig. 1f).

\section{Treatment of the specimens}

\section{Fracturing of the vertebral bodies and implantation}

To achieve a reproducible fracture pattern corresponding to Type A3 burst fracture [2], the vertebral body was weakened by compressing the cancellous bone via a hole in the lateral surface. The cortices otherwise were left intact. The fracture was then induced by asymmetric (anterior) compression in a material testing machine (Zwick 1454, Zwick GmbH \& Co. $\mathrm{KG}$, Ulm, Germany). A continuously increasing force (feed rate: $10 \mathrm{~mm} / \mathrm{min}$ ) was applied until the anterior wall of the vertebral body was reduced to half of its original height. This corresponds to the average height loss in A3 burst fractures [3].

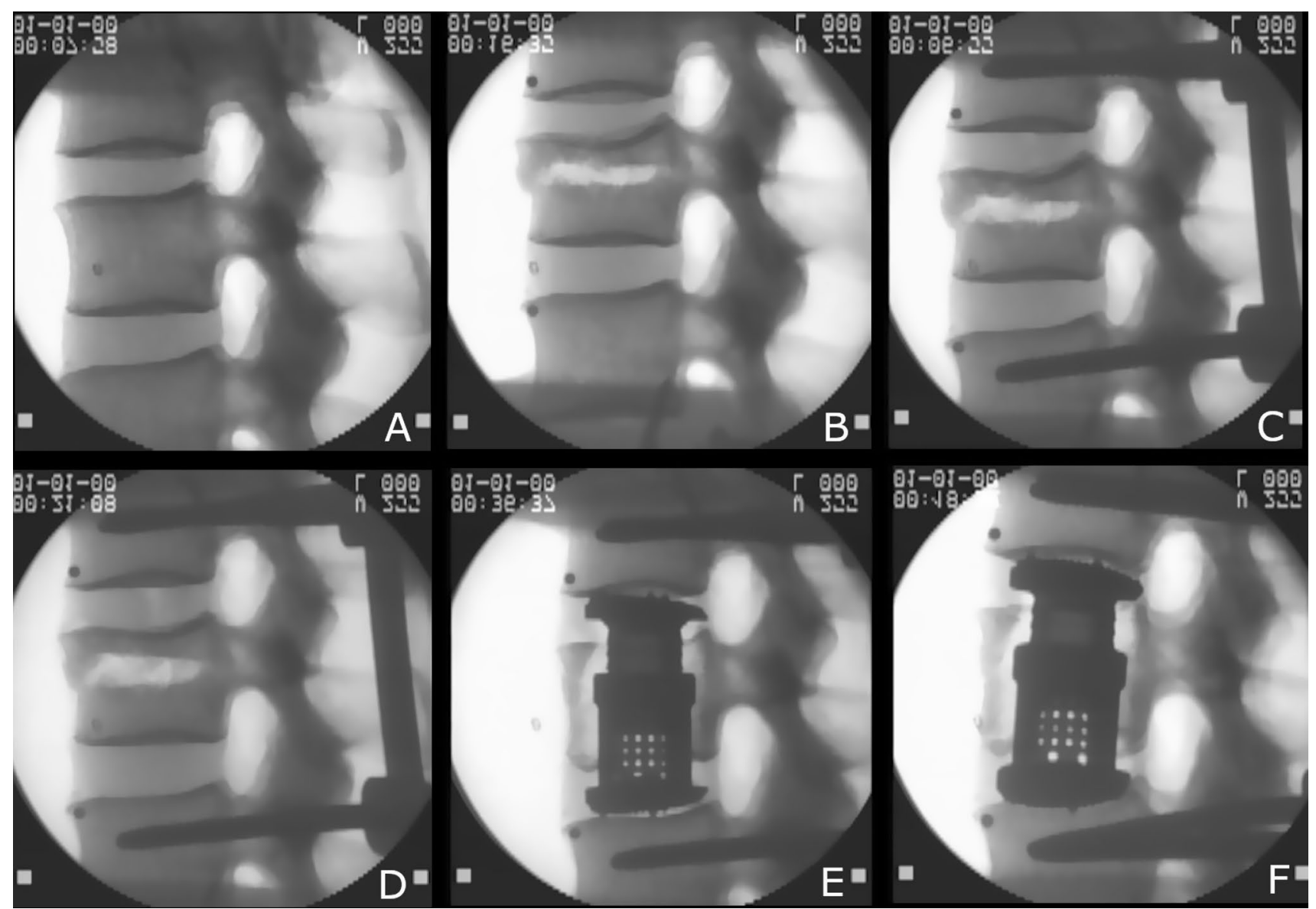

Fig. 1 Lateral fluoroscopy of the intact specimen (a), of the fractured specimen (b) of the specimen instrumented in neutral position (c), of the specimen instrumented in distraction (d), of the specimen with a cage and posterior instrumentation in distraction (e), and specimen with a cage and posterior instrumentation in compression (f). Note the change of Cobb`s angle that occurs over the different conditions of the set-up 
Lateral $\mathrm{x}$-rays have been taken after the injury. They showed a fracture of the posterior wall with bulging into the spinal canal.

Posterior instrumentation of the vertebra above the fracture and to the vertebra below the fracture was performed in a standardized manner using the USS instrumentation (USS Fracture System, Synthes GmbH, Oberdorf, Switzerland). Instrumentation was fixed with restoration of the height of the fractured vertebral body by lordosation without applying compressive or distractive force.

For anterior stabilization, a standard expandable cage was used (Synex cage $10^{\circ}$, Synthes GmbH, Oberdorf, Switzerland). The fractured vertebral body was opened from the left side, partially removed; the adjacent discs were incised and removed completely. Anterior and posterior longitudinal ligaments were preserved. The expandable cage was inserted centrally and distracted until the anterior ligament was tight, and the height of the vertebral body was restored.

\section{Statistics}

Statistical analysis was performed using SPSS 27 (IBM Corp., Armonk, NY, United States). Since only $n=6$ specimens were tested, a normal distribution of the data cannot be assumed. For that reason, median values with ranges are presented and non-parametric tests were used for the statistical evaluation.

In order to proof our hypothesis, only the two circumferential conditions with distraction and compression were analysed using the Wilcoxon Signed Rank Test in order to compare ROM, NZ, and also the values of sagittal angle. The significance level was set to 0.05 .

\section{Results}

\section{Range of motion and neutral zone}

ROM as well as NZ increased after induction of the fracture in the vertebral body in all three motion planes (Figs. 2,
Fig. 2 Bar graph giving median/ (coloured) and NZ (grey) in flexion/extension maximum-minimum for ROM

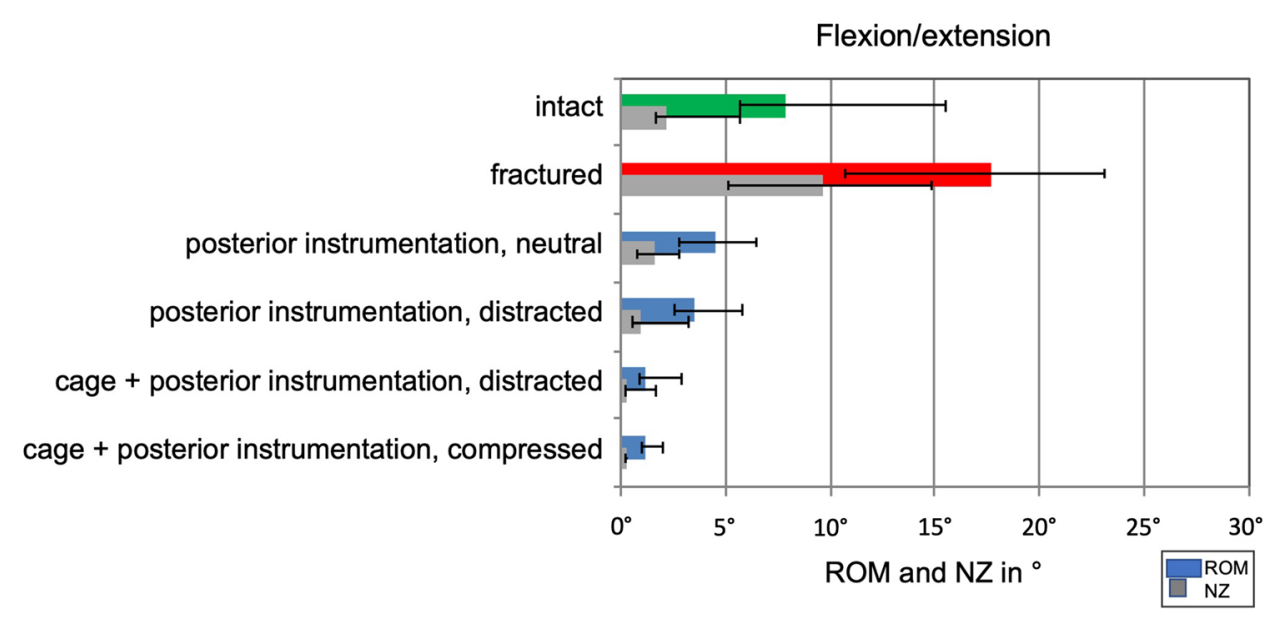

Fig. 3 Bar graph giving median/ maximum-minimum for ROM (coloured) and NZ (grey) in right/left lateral bending

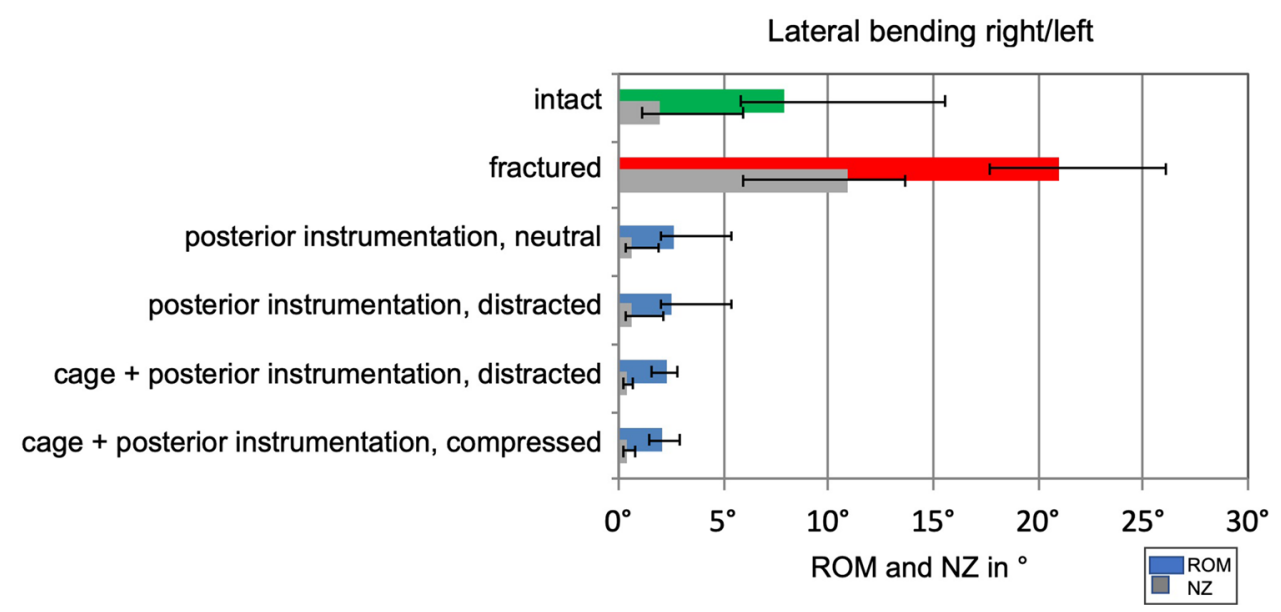


Fig. 4 Bar graph giving median/ maximum-minimum for ROM (coloured) and NZ (grey) left/ right axial rotation. Statistically significant differences are indicated by asterisks

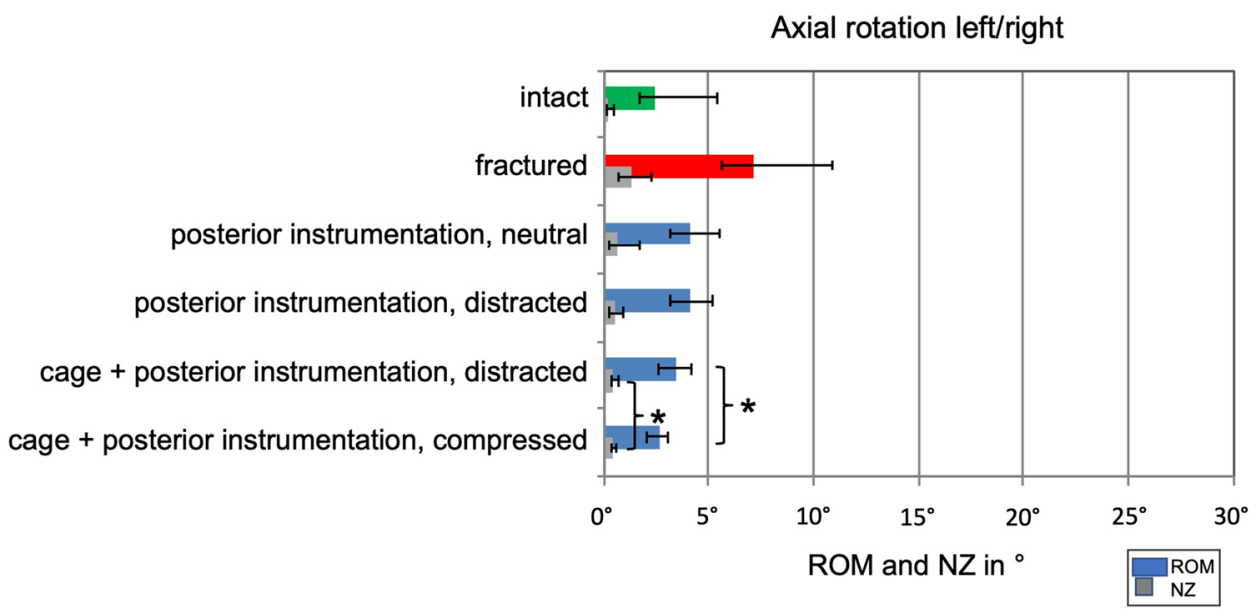

3 and 4, Table 1). The posterior instrumentation, in neutral or distracted position, was able to stabilize the instrumented segment compared to the fractured condition in all three movement axes. When compared to the intact spine, the instrumentation decreased the flexibility markedly in flexion/extension as well as lateral bending; however, the instrumentation was not able to decrease flexibility below the intact state in axial rotation (Figs. 2, 3 and 4, Table 1).

The insertion of a cage was able to further enhance stability particularly in flexion/ extension.

The last step, additional posterior compression via the instrumentation, statistically significantly decreased ROM and NZ in axial rotation compared to the distracted condition and reached values of the intact spine (Fig. 4, Table 1). Circumferential stabilization consisting of an anterior cage in combination with a posterior compression instrumentation was the only construct that was able to restore stability of the injured segment in axial rotation comparable to the intact specimen.

In flexion/extension and lateral bending flexibility of the both circumferential stabilized conditions are comparable (Figs. 2 and 3, Table 1).

\section{Sagittal profile}

The median of sagittal Cobb angle in the intact specimens was lordotic with $-3.3^{\circ}$. After fracturing of the vertebral body, the lordosis angle decreases to $-0.8^{\circ}$, and increases again to $-1.7^{\circ}$ following posterior instrumentation. Distracting the instrumentation changes the median Cobb angle in a kyphotic position to $+1.1^{\circ}$. Insertion of the cage with anterior distraction changes the angle to lordosis again $\left(-2.2^{\circ}\right)$. Posterior final compression via pedicle screws resulted again in a drastic increase in lordosis $\left(-8.4^{\circ}\right)$ when compared to the neutral or even distracted situation.

Regarding the both circumferential stabilized states, the increase in bisegmental Cobb angle was statistically significant ( $p=0.043$ ) between the distracted position and the compressed position of the posterior instrumentation (Fig. 5).

\section{Discussion}

Compression fractures at the thoracolumbar junction are the most common fractures within the TL spine. Despite the frequency of the injury, there is no generally accepted treatment recommendation up to now [19]. According to the second, internet-based multicenter study of the Spine Study Group of the German Association of Trauma Surgery, 47\% of the patients are treated with a single posterior instrumentation, $47 \%$ with a posterior instrumentation combined with an additional anterior support, and $6 \%$ with a single anterior approach. Removal of the implants was performed in $75 \%$ of the patients after median time of 12 months [1].

A principal problem in the treatment of TL fractures is the loss of correction during follow-up. With single posterior instrumentation, an average loss of correction of $6.25^{\circ}$ was found [1]. Further studies support these finding going along with a high failure rate of the implants [20-23]. A combined posterior-anterior procedure provided a better maintenance of the correction compared to the posterior only group [9]; however, even after this more complex surgical procedure there was a marked loss of correction of average $3.6^{\circ}[1]$.

Loss of correction is related with an inferior clinical result; increasing kyphosis was seen to be an important factor to impair quality of life after surgical treatment of fractures at the thoracolumbar junction [24].

To overcome the problem of secondary hyperkyphosis, it would be beneficial to improve the initial correction and to achieve a quick and reliable bony fusion. Precondition for a rapid fusion is a high initial stability of the instrumentation in all movement axes, the most critical movement axis with respect to stabilization being the axial rotation [12-14]. 
Table 1 Single values of total $\mathrm{ROM}\left(^{\circ}\right)$ and $\mathrm{NZ}\left(^{\circ}\right)$ of each specimen in the three motion planes

\begin{tabular}{|c|c|c|c|c|c|c|c|}
\hline & $\# 1(69 / \mathrm{m})$ & \#2 (77/m) & \#3 (56/m) & \#4 (58/f) & \#5 (56/m) & \#6 (59/f) & $p$ \\
\hline \multicolumn{8}{|l|}{ Flexion/extension } \\
\hline ROM intact & 5.8 & 5.6 & 7.4 & 15.5 & 7.8 & 8.4 & \\
\hline ROM fractured & 10.7 & 14.7 & 17.1 & 23.1 & 19.8 & 18.3 & \\
\hline ROM post. instr. neutral & 6.4 & 5.7 & 2.8 & 3.6 & 4.3 & 4.6 & \\
\hline ROM post. instr. distracted & 5.8 & 3.9 & 2.7 & 2.5 & 3.7 & 3.3 & \\
\hline ROM cage + post. instr. distracted & 2.0 & 2.9 & 0.9 & 1.1 & 1.2 & 0.8 & 0.463 \\
\hline ROM cage + post. instr. compressed & 1.5 & 2.0 & 0.9 & 1.2 & 1.1 & 0.9 & \\
\hline NZ intact & 1.6 & 1.8 & 1.9 & 5.7 & 2.5 & 2.9 & \\
\hline $\mathrm{NZ}$ fractured & 5.0 & 9.0 & 8.1 & 14.8 & 12.6 & 10.3 & \\
\hline $\mathrm{NZ}$ post. instr. neutral & 2.8 & 2.2 & 0.8 & 1.0 & 1.5 & 1.6 & \\
\hline $\mathrm{NZ}$ post. instr. distracted & 3.2 & 1.1 & 0.8 & 0.6 & 1.1 & 0.5 & \\
\hline $\mathrm{NZ}$ cage + post. instr. distracted & 0.5 & 1.6 & 0.2 & 0.3 & 0.3 & 0.3 & 0.249 \\
\hline $\mathrm{NZ}$ cage + post. instr. compressed & 0.4 & 0.6 & 0.3 & 0.2 & 0.2 & 0.2 & \\
\hline \multicolumn{8}{|l|}{ Lateral bending } \\
\hline ROM intact & 7.7 & 5.8 & 8.1 & 15.6 & 7.3 & 10.1 & \\
\hline ROM fractured & 17.6 & 18.0 & 19.2 & 25.8 & 26.1 & 22.8 & \\
\hline ROM post. instr. neutral & 5.4 & 3.0 & 2.1 & 2.2 & 4.1 & 2.0 & \\
\hline ROM post. instr. distracted & 5.4 & 2.9 & 2.0 & 2.2 & 4.1 & 2.1 & \\
\hline ROM cage + post. instr. distracted & 2.8 & 2.8 & 1.6 & 1.9 & 2.8 & 1.6 & 0.345 \\
\hline ROM cage + post. instr. compressed & 2.9 & 2.7 & 1.4 & 1.8 & 2.3 & 1.7 & \\
\hline $\mathrm{NZ}$ intact & 1.8 & 1.1 & 2.1 & 5.9 & 1.5 & 3.3 & \\
\hline NZ fractured & 6.0 & 7.4 & 9.3 & 13.2 & 13.6 & 12.7 & \\
\hline $\mathrm{NZ}$ post. instr. neutral & 2.0 & 0.7 & 0.4 & 0.4 & 1.1 & 0.5 & \\
\hline $\mathrm{NZ}$ post. instr. distracted & 2.1 & 0.7 & 0.4 & 0.4 & 1.0 & 0.6 & \\
\hline $\mathrm{NZ}$ cage + post. instr. distracted & 0.7 & 0.6 & 0.3 & 0.3 & 0.6 & 0.3 & 0.249 \\
\hline $\mathrm{NZ}$ cage + post. instr. compressed & 0.8 & 0.8 & 0.3 & 0.3 & 0.6 & 0.3 & \\
\hline \multicolumn{8}{|l|}{ Axial rotation } \\
\hline ROM intact & 2.6 & 1.7 & 2.4 & 5.4 & 3.4 & 2.4 & \\
\hline ROM fractured & 5.8 & 7.5 & 5.6 & 10.8 & 8.6 & 6.8 & \\
\hline ROM post. instr. neutral & 5.5 & 4.0 & 3.1 & 4.2 & 4.5 & 4.0 & \\
\hline ROM post. instr. distracted & 5.2 & 3.8 & 3.2 & 4.2 & 4.4 & 4.0 & \\
\hline ROM cage + post. instr. distracted & 3.6 & 4.2 & 2.8 & 3.4 & 3.8 & 2.6 & $\mathbf{0 . 0 2 8}$ \\
\hline ROM cage + post. instr. compressed & 2.6 & 2.8 & 2.1 & 2.8 & 3.1 & 2.3 & \\
\hline NZ intact & 0.3 & 0.1 & 0.2 & 0.5 & 0.3 & 0.2 & \\
\hline $\mathrm{NZ}$ fractured & 1.0 & 2.0 & 0.7 & 2.3 & 1.5 & 1.3 & \\
\hline NZ post. instr. neutral & 1.7 & 0.6 & 0.3 & 0.5 & 0.8 & 0.6 & \\
\hline $\mathrm{NZ}$ post. instr. distracted & 1.0 & 0.5 & 0.3 & 0.5 & 0.6 & 0.5 & \\
\hline $\mathrm{NZ}$ cage + post. instr. distracted & 0.7 & 0.5 & 0.4 & 0.4 & 0.7 & 0.4 & $\mathbf{0 . 0 2 8}$ \\
\hline $\mathrm{NZ}$ cage + post. instr. compressed & 0.5 & 0.4 & 0.4 & 0.4 & 0.6 & 0.3 & \\
\hline
\end{tabular}

$p$ values $<0.05$ are printed in bold

In this biomechanical study, a Type A fracture within the first lumbar vertebra was created in vitro in a standardized manner. Stepwise application of a routine posterior instrumentation and a cage was performed. We found that all posterior instrumentations significantly stabilized the treated segment in all three loading cases; for flexion/extension and lateral bending stability was superior to the intact state, however, inferior in axial rotation. A supplementary anterior cage significantly increased the stability in flexion/ extension, but again failed to provide sufficient stability in axial rotation. Thus, axial rotation is the critical loading case in this scenario that potentially could produce implant failure or loss of correction in patients. Solely the construct consisting of a posterior instrumentation fixed under compression in combination with an anterior cage regained stability in axial rotation comparable to the intact spine. The effects 
Fig. 5 Bar graph, giving median/ranges for bisegmental Cobb`s angle of the injured/ treated segment. Statistically significant differences are indicated by asterisks. Kyphosis corresponds to + and lordosis corresponds to -

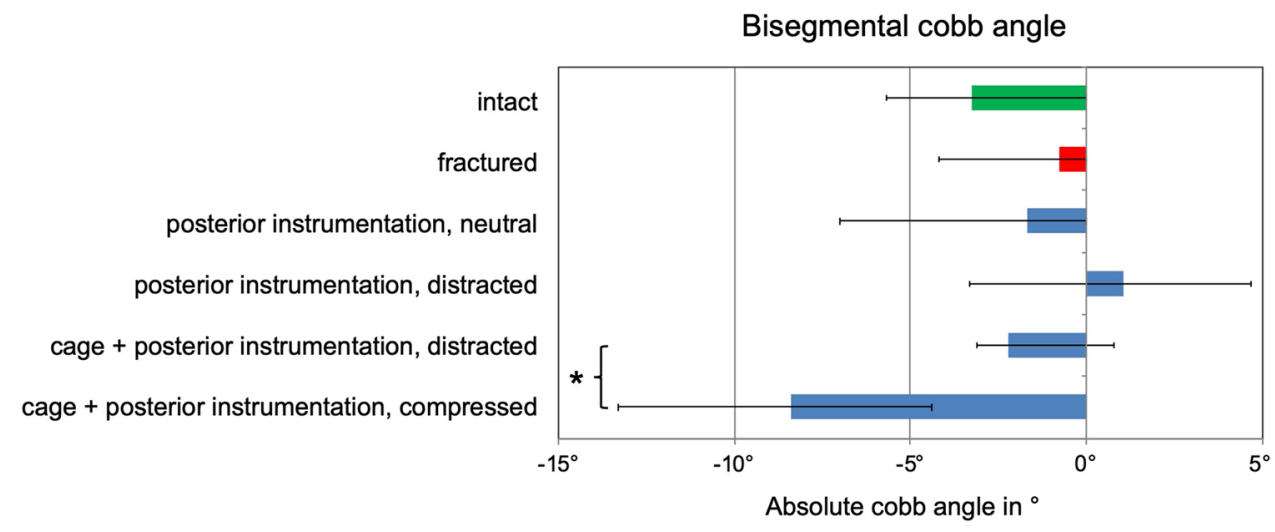

of compression versus distraction in flexion/extension and lateral bending are small and not significant. However, in axial rotation, significant differences were seen. Thus, final compression of the posterior instrumentation as the last step may be considered to give a relevant "plus" in stability and to optimize initial stability.

The increase in the rotational stability by posterior compression may be explained by a locking of the joint facets under compression. In contrast to the thoracic spine, the orientation of the facet joints in the upper lumbar spine changes to a more sagittal direction [25], thus controlling predominantly rotation. It also may be anticipated, that the anterior column-although not measured-is set under compression, thus promoting stability and loading of the cage. Both, loading of the cage and locking the facets may promote fusion in clinical scenarios.

Furthermore, the additional posterior compression via the instrumentation improved the initial correction of the kyphotic deformity. Posterior compression against the cage acting as a pivot increased the lordosis angle compared to all other conditions. The increase in lordosis was statistically significant. The lordotic effect can be seen by the divergence of the shafts of the pedicle screws (Fig. 1f).

Some limitations within this study should be noted: It seems that realistic loading conditions would mean to simulate body weight, which can be done with preload or a so-called follower load. However, this often leads to unwanted artefacts like coupled motion, which cannot be well controlled. Therefore, internationally accepted recommendations suggest to use pure moments without preload [16]. This leads to very standardized conditions, and this concept with pure moments can be replicated in different ways from other groups, which leads to very comparable results. Furthermore, it has been proven, that the application of pure moments to intact lumbar spinal specimens in vitro produces forces and moments in implants comparable with loads observed in vivo [26]. Therefore, it seems that this selected test set-up is the best possible compromise for this experiment.
The recommendations also suggest to apply pure moment with $7.5 \mathrm{Nm}$, but allow to reduce them to 3.75 $\mathrm{Nm}$ if necessary [16]. Therefore, a preliminary test was performed before finalizing the testing protocol. In this preliminary test one specimen was lost, because it was destroyed in the state after fracture with a moment up to $7.5 \mathrm{Nm}$, which suggested to us $3.75 \mathrm{Nm}$ instead.

Next, our results presented here are only applicable to immediate post-operative fixation and do not include the effects of cyclic loading. As a consequence, we are not able to give long term predictions with respect to stability, fusion tendency, and sagittal profile. Nevertheless, a "plus" in stability by blocking of the facets may help to avoid screw loosening and promote fusion.

Third, the influence of the muscular envelope, fascia and skin is completely neglected. This is important, because the influence of these soft tissues, applying posterior tension to the human spine, cannot be overemphasized.

Lateral $\mathrm{x}$-rays have been taken after the injury for each specimen. Here, posterior wall fractures could be visualized in each, however, not documented via CAT scans. TL compression fractures Type A3 may be present with different types of posterior wall destruction. This aspect has not been investigated in the current study, due to the impossibility to create a constant destruction of the posterior wall. It must be noted, that the results may be influenced by the degree of posterior wall destruction as well as by posterior wall removal.

Finally, direct transfer of biomechanical results to clinical problems is difficult since we do not know the optimum degree of stabilization required for healing. Also, a lot of other factors (age, bone density, degree of degeneration, type of trauma, the absence of muscular, and skin envelope, etc.) may and will differ between this in vitro scenario and real-life conditions. These aspects limit the final conclusions of our study, but this is true for every in vitro set-up. However, our results highlight the importance of the posterior compression to the facet joints via the applied pedicle screws. 
Facing the above-mentioned limits of the current study, it must be looked at its unique design, which, to the best of our knowledge illuminates the importance of posterior compression as the last step in TL fracture surgery in the way described. Moreover, we describe a standardized model for simulation of thoracolumbar compression fractures.

The authors could show that this final compression results in significant increase in segmental stability and significantly improves the sagittal profile within the TL junction compared to the instrumentation in distraction. To transfer these aspects into surgical scenarios, we recommend applying the following strategies for situations like mentioned above: Start surgery via posterior approach, apply posterior instrumentation, and perform decompression if needed. Next, change to a standard anterior thoracic or thoracolumbar anterior approach. Resect the vertebral body and replace it by an appropriate implant, filled with bone or place bone around the implant.

In case that the posterior instrumentation is not fixed in compression: Complete surgery by posterior approach; compress the treated segments using compression forcipes before the nuts are tightened. In conclusion, do not finish surgery for these pathologies without posterior compression to lock the facet joints and to increase lordosis.

Clinical consequence, transferred from this in vitro setting to clinical activity, may be: Surgeons should consider final posterior compression of the instrumentation as the very last step in spinal fixation of pathologies within the TL junction that are treated via vertebral body replacement and posterior instrumentation. This final compression could help to add some more stability and to improve physiological alignment within this region thus helping to overcome impairment of the alignment within the postoperative course.

Acknowledgements The authors would like to dedicate the paper to Prof. Dr. J. Harms, who hypothesized the beneficial effects of final posterior compression in spine surgery.

Authors' contributions MR contributed to idea, preparation of the manuscript. TP contributed to preparation of the manuscript. IN contributed to specimen preparation, testing. DV contributed to specimen preparation, testing, statistics. JD contributed to preparation of the manuscript. KP contributed to donation of the specimen. HJW contributed to testing, statistics, preparation of the manuscript.

Funding Open Access funding enabled and organized by Projekt DEAL. This study was supported by the German Spine Foundation. Donation of implants and instruments was used within the study by DePuy Synthes.

Data availability Data stored at the Institute of Orthopaedic Research and Biomechanics, Ulm, Germany.

Code availability Software application or custom code.

\section{Declarations}

Conflict of interest The author's declared that they have no conflict of interest.

Ethical approval The study was approved by the ethics committee of the University of Ulm (No. 46/13).

Open Access This article is licensed under a Creative Commons Attribution 4.0 International License, which permits use, sharing, adaptation, distribution and reproduction in any medium or format, as long as you give appropriate credit to the original author(s) and the source, provide a link to the Creative Commons licence, and indicate if changes were made. The images or other third party material in this article are included in the article's Creative Commons licence, unless indicated otherwise in a credit line to the material. If material is not included in the article's Creative Commons licence and your intended use is not permitted by statutory regulation or exceeds the permitted use, you will need to obtain permission directly from the copyright holder. To view a copy of this licence, visit http://creativecommons.org/licenses/by/4.0/.

\section{References}

1. Reinhold M, Knop C, Beisse R, Audige L, Kandziora F, Pizanis A, Pranzl R, Gercek E, Schultheiss M, Weckbach A, Buhren V, Blauth M (2010) Operative treatment of 733 patients with acute thoracolumbar spinal injuries: comprehensive results from the second, prospective, internet-based multicenter study of the Spine study group of the German association of trauma surgery. Eur Spine J 19(10):1657-1676. https://doi.org/10.1007/ s00586-010-1451-5

2. Magerl F, Aebi M, Gertzbein SD, Harms J, Nazarian S (1994) A comprehensive classification of thoracic and lumbar injuries. Eur Spine J 3(4):184-201. https://doi.org/10.1007/BF02221591

3. Payer M (2006) Unstable burst fractures of the thoraco-lumbar junction: treatment by posterior bisegmental correction/fixation and staged anterior corpectomy and titanium cage implantation. Acta Neurochir (Wien) 148(3):299-306. https://doi.org/10.1007/ s00701-005-0681-5

4. Salas N, Prebet R, Guenoun B, Gayet LE, Pries P (2011) Vertebral body cage use in thoracolumbar fractures: outcomes in a prospective series of 23 cases at 2 years' follow-up. Orthop Traumatol Surg Res 97(6):602-607. https://doi.org/10.1016/j.otsr.2011.05. 003

5. Vaccaro AR, Lim MR, Hurlbert RJ, Lehman RA, Jr, Harrop J, Fisher DC, Dvorak M, Anderson DG, Zeiller SC, Lee JY, Fehlings MG, Oner FC, Spine Trauma Study G (2006) Surgical decision making for unstable thoracolumbar spine injuries: results of a consensus panel review by the Spine trauma study group. J Spinal Disord Tech 19(1):1-10. https://doi.org/10.1097/01.bsd.00001 80080.59559 .45

6. Keynan O, Fisher CG, Vaccaro A, Fehlings MG, Oner FC, Dietz J, Kwon B, Rampersaud R, Bono C, France J, Dvorak M (2006) Radiographic measurement parameters in thoracolumbar fractures: a systematic review and consensus statement of the spine trauma study group. Spine 31(5):E156-165. https://doi.org/10. 1097/01.brs.0000201261.94907.0d

7. Merkel P, Hauck S, Zentz F, Buhren V, Beisse R (2008) Spinal column injuries in sport: treatment strategies and clinical results. Unfallchirurg 111(9):711-718. https://doi.org/10.1007/ s00113-008-1456-2 
8. Oprel PP, Tuinebreijer WE, Patka P, den Hartog D (2010) Combined anterior-posterior surgery versus posterior surgery for thoracolumbar burst fractures: a systematic review of the literature. Open Orthop J 4:93-100. https://doi.org/10.2174/1874325001 004010093

9. Scholz M, Kandziora F, Tschauder T, Kremer M, Pingel A (2018) Prospective randomized controlled comparison of posterior versus posterior-anterior stabilization of thoracolumbar incomplete cranial burst fractures in neurological intact patients: the RASPUTHINE pilot study. Eur Spine J 27(12):3016-3024. https://doi.org/10.1007/s00586-017-5356-4

10. Mueller LA, Mueller LP, Schmidt R, Forst R, Rudig L (2006) The phenomenon and efficiency of ligamentotaxis after dorsal stabilization of thoracolumbar burst fractures. Arch Orthop Trauma Surg 126(6):364-368. https://doi.org/10.1007/s00402-005-0065-6

11. Eysel P, Hopf C, Furderer S (2001) Kyphotic deformation in fractures of the thoracic and lumbar spine. Orthopade 30(12):955964. https://doi.org/10.1007/s001320170009

12. Bishop FS, Samuelson MM, Finn MA, Bachus KN, Brodke DS, Schmidt MH (2010) The biomechanical contribution of varying posterior constructs following anterior thoracolumbar corpectomy and reconstruction. J Neurosurg Spine 13(2):234-239. https://doi. org/10.3171/2010.3.SPINE09267

13. Viljoen SV, DeVries Watson NA, Grosland NM, Torner J, Dalm B, Hitchon PW (2014) Biomechanical analysis of anterior versus posterior instrumentation following a thoracolumbar corpectomy: laboratory investigation. J Neurosurg Spine 21(4):577-581. https://doi.org/10.3171/2014.6.SPINE13751

14. Slosar PJ Jr, Patwardhan AG, Lorenz M, Havey R, Sartori M (1995) Instability of the lumbar burst fracture and limitations of transpedicular instrumentation. Spine 20(13):1452-1461. https:// doi.org/10.1097/00007632-199507000-00003

15. Stoltze D, Harms J (1999) Correction of traumatic deformities. Princ Tech Orthopade 28(8):731-745. https://doi.org/10.1007/ PL00003661

16. Wilke HJ, Wenger K, Claes L (1998) Testing criteria for spinal implants: recommendations for the standardization of in vitro stability testing of spinal implants. Eur Spine J 7(2):148-154

17. Wilke H-J, Jungkunz B, Wenger K, Claes LE (1998) Spinal segment range of motion as a function of in vitro test conditions: effects of exposure period, accumulated cycles, angular-deformation rate, and moisture condition. Anat Rec 251(1):15-19

18. Wilke HJ, Claes L, Schmitt H, Wolf S (1994) A universal spine tester for in vitro experiments with muscle force simulation. Eur Spine J 3(2):91-97

19. Verheyden AP, Spiegl UJ, Ekkerlein H, Gercek E, Hauck S, Josten C, Kandziora F, Katscher S, Kobbe P, Knop C, Lehmann W,
Meffert RH, Muller CW, Partenheimer A, Schinkel C, Schleicher P, Scholz M, Ulrich C, Hoelzl A (2018) Treatment of fractures of the thoracolumbar Spine: recommendations of the Spine section of the german society for orthopaedics and trauma (DGOU). Global Spine J 8(2 Suppl):34S-45S. https://doi.org/10.1177/21925 68218771668

20. Xu BS, Tang TS, Yang HL (2009) Long-term results of thoracolumbar and lumbar burst fractures after short-segment pedicle instrumentation, with special reference to implant failure and correction loss. Orthop Surg 1(2):85-93. https://doi.org/10.1111/j. 1757-7861.2009.00022.x

21. Martiniani M, Vanacore F, Meco L, Specchia N (2013) Is posterior fixation alone effective to prevent the late kyphosis after T-L fracture? Eur Spine J 22(Suppl 6):S951-56. https://doi.org/ 10.1007/s00586-013-3008-x

22. Cavanaugh D, Usmani MF, Weir TB, Camacho J, Yousaf I, Khatri V, Bivona L, Shasti M, Koh EY, Banagan KE, Ludwig SC, Gelb DE (2020) Radiographic evaluation of minimally invasive instrumentation and fusion for treating unstable spinal column injuries. Global Spine J 10(2):169-176. https://doi.org/10.1177/21925 68219856872

23. Gelb D, Ludwig S, Karp JE, Chung EH, Werner C, Kim T, Poelstra K (2010) Successful treatment of thoracolumbar fractures with short-segment pedicle instrumentation. J Spin Disord Tech 23(5):293-301. https://doi.org/10.1097/BSD.0b013e3181af20b6

24. Schulz R, Melcher RP, Garib MC, Schulz H, Weissman K, Harms J (2014) Does kyphotic deformity correlate with functional outcomes in fractures at the thoracolumbar junction treated by 360 degrees instrumented fusion? Eur J Orthop Surg Traumatol 24(Suppl 1):S93-101. https://doi.org/10.1007/s00590-014-1435-y

25. Masharawi Y, Rothschild B, Dar G, Peleg S, Robinson D, Been E, Hershkovitz I (2004) Facet orientation in the thoracolumbar spine: three-dimensional anatomic and biomechanical analysis. Spine 29(16):1755-1763. https://doi.org/10.1097/01.brs.0000134575. 04084.ef

26. Wilke HJ, Rohlmann A, Neller S, Schultheiss M, Bergmann G, Graichen F, Claes LE (2001) Is it possible to simulate physiologic loading conditions by applying pure moments? A comparison of in vivo and in vitro load components in an internal fixator. Spine 26(6):636-642. https://doi.org/10.1097/00007632-20010 3150-00014

Publisher's Note Springer Nature remains neutral with regard to jurisdictional claims in published maps and institutional affiliations. 